\title{
ROJ
}

\section{Effectiveness of radiotherapy for head and neck skin cancers: a single-institution study}

\author{
Jae Wang Kim², Byung Min Yun², Myoung Soo Shin², Jae Kyoung Kang ${ }^{2}$, JungJu Kim³, Young Suk Kim ${ }^{4}$ \\ 'Department of Dermatology, Jeju National University School of Medicine, Jeju National University Hospital, Jeju, Korea \\ ${ }^{2}$ Department of Plastic and Reconstructive Surgery, Jeju National University School of Medicine, Jeju National University Hospital, \\ Jeju, Korea \\ ${ }^{3}$ Medical Course, Jeju National University School of Medicine, Jeju, Korea \\ ${ }^{4}$ Department of Radiation Oncology, Jeju National University School of Medicine, Jeju National University Hospital, Jeju, Korea
}

Purpose: External beam radiotherapy (EBRT) is a useful option to treat head and neck skin cancer patients who are not indicated for surgery. In this study, we evaluated the treatment outcomes of EBRT in an Asian population.

Materials and Methods: The records from 19 head and neck skin cancer patients (10 with squamous cell carcinoma and 9 with basal cell carcinoma) who were treated with definitive or adjuvant EBRT from 2009 to 2017 were retrospectively reviewed. The radiotherapy doses administered ranged from 50 to $66 \mathrm{~Gy}$ (median, $55 \mathrm{~Gy}$ ) with 2.0-2.75 Gy per daily fraction (median, $2.5 \mathrm{~Gy}$ ). The T stage at presentation was as follows: Tis (1 patient), T1 (11 patients), T2 (6 patients), and T3 (1 patient). None had regional lymph node disease or distant metastasis at presentation. The local failure-free survival (LFFS) rates, toxicity, and cosmetic results were analyzed.

Results: The median age was 75.5 years (range, 52.6 to 92.5 years). The median follow-up duration from the completion of radiotherapy was 44.9 months (range, 5.8 to 82.6 months). One local failure occurred in a patient with a 2.1-cm posterior neck squamous cell carcinoma at 32.5 months after radiotherapy $(1 / 19,5.3 \%)$. The 3 -year LFFS rate was $91.7 \%$. No patients died from skin cancer during follow-up, and no grade 3 complications occurred. The cosmetic outcomes were excellent for 16 (84.2\%) and good for 3 (15.8\%) of the 19 patients.

Conclusion: EBRT offers good local control and cosmetic outcomes in patients with head and neck skin cancer, with no grade 3 complications.

Keywords: Skin cancer, Radiotherapy, Treatment outcome, Toxicity

\section{Introduction}

Non-melanoma skin cancer is the single most common cancer in the United States [1]. Most cases are basal cell carcinoma (BCC) and squamous cell carcinoma (SCC) [2].
Although the incidence is significantly lower in the Asian population than in the Caucasian population [3], this cancer type has recently become more common in Korea, probably because of the depletion of the ozone layer, increased use of immunosuppressive drugs, and the increasing age and

Received 12 July 2019, Revised 10 September 2019, Accepted 30 September 2019.

Correspondence: Young Suk Kim, Department of Radiation Oncology, Jeju National University Hospital, Jeju National University School of Medicine, 15 Aran 13-gil, Jeju 63241, Korea. Tel: +82-64-717-1330, Fax: +82-64-717-1169, E-mail: yskim@ jejunuh.co.kr (http://orcid.org/0000-0002-0640-1679)

(c) This is an Open Access article distributed under the terms of the Creative Commons Attribution Non-Commercial License (http://creativecommons.org/ licenses/by-nc/4.0/) which permits unrestricted non-commercial use, distribution, and reproduction in any medium, provided the original work is properly cited.

www.e-roj.org 
increased sun exposure of the population [3].

Surgical excision is the most frequently used treatment modality for basal and squamous cell skin cancers and is considered the treatment of choice for most tumors [4]. The most frequent site of this tumor is the face, where radical excision is limited by the proximity of essential anatomic structures [5]. The recommendation of definitive external beam radiotherapy (EBRT) is often made in patients where the cosmetic and/or functional outcomes would be better with radiotherapy than with surgery according to the size and location of the tumor [6]. EBRT is also a useful option for treatment in elderly patients with multiple comorbidities who are not indicated for surgery [7].

In contrast to most European, Australian, and North American (i.e., Caucasian) countries, reports of radiotherapy use for treatment of basal and squamous cell skin cancers in Asian countries are rare [8]. The aim of the present study was to analyze the local failure-free survival (LFFS) rate, toxicity, and cosmetic outcomes of EBRT for basal and squamous cell skin cancers in the head and neck regions in an Asian population.

\section{Materials and Methods}

\section{Patients}

The medical records of 232 patients with skin cancer who were treated at our institution between August 2009 and September 2017 were retrospectively reviewed. Institutional Review Board of Jeju National University Hospital (No. 201806-001) approved this study. Twenty-six of 232 patients (11.2\%) were treated with EBRT. Seven patients were excluded from the analysis; of these, 1 patient was diagnosed with Merkel cell carcinoma, 1 patient was diagnosed with malignant melanoma, 1 patient refused the completion of scheduled radiotherapy after its initiation, 2 patients received EBRT with palliative intent, 1 patient had SCC on the foot, and 1 patient had SCC on the chest wall. Thus, the data from 19 patients were analyzed. Pretreatment biopsy was performed to confirm the diagnosis. High-risk features such as tumor grade, the presence of perineural invasion, or depth of invasion could not be consistently assigned to most patients due to limited pathological data from biopsies [9].

All the patients were examined by a dermatologist and a plastic surgeon before radiotherapy evaluation, who had ruled out the feasibility or appropriateness of surgical resection or other local treatments [10]. In general, patients who were considered operable to achieve adequate excision and maintain organ functions proceeded to surgery [9]. EBRT was recommended in patients who declined surgery, were elderly with comorbidities, were expected to show a poor cosmetic outcome with surgery, and/or were of poor performance status [9]. The Eastern Cooperative Oncology Group performance status was recorded. A series of digital photographs of the initial lesion on each patient was obtained [11].

Diagnostic magnetic resonance imaging (MRI) and computed tomography (CT) with contrast medium were performed in $2(10.5 \%)$ and 6 (31.6\%) patients, respectively, to assess the disease extent, including the depth of invasion before the start of EBRT. For multifocal tumors, the diameter of the largest tumor was considered the primary tumor size. Clinical staging was determined according to the American Joint Committee on Cancer staging system, 7th edition.

\section{Treatment}

The planning CT scans were obtained with or without contrast medium with a slice thickness of 3-5 mm using a 16-slice CT scanner (Brilliance CT Big Bore; Philips Medical Systems, Cleveland, OH, USA) in all patients. Twelve patients (63.2\%) were treated using thermoplastic immobilization masks to ensure adequate immobilization during therapy and reproducibility. Palpation of the skin cancer edges was carried out to detect any areas of induration, indicating cancer spread not visible on inspection, and a thin wire was placed to outline the areas clinically involved by the cancer [12]. Treatment was conducted using a linear accelerator with the Millennium 120leaf multi-leaf collimator system (Clinac iX; Varian Medical Systems Inc., Palo Alto, CA, USA). Patient setup was verified weekly by $\mathrm{kV}$ portal images obtained using the On-Board Imager system prior to treatment. None of the patients were treated with brachytherapy.

The gross tumor volume was assessed visually, and a CT scan was used to assess skin depth and the depth of invasion before treatment [13]. A 1.5- to 3-cm margin was added to the gross tumor volume for microscopic extension (clinical target volume), and an additional $0.2-$ to $0.5-\mathrm{cm}$ margin was added for setup uncertainty (planning target volume). In 7 patients (36.8\%), cone down boost fields (defined as a $1-$ to $2-\mathrm{cm}$ margin added to the gross tumor volume) with an additional 0.2- to 0.5-cm margin (planning target volume) were applied at the median dose of $50 \mathrm{~Gy}$ (range, 40 to $54 \mathrm{~Gy}$ ). A smaller treatment margin was accepted in certain anatomical locations such as the tip of the nose, ala nasi, and facial areas near the eye [14]. Three-dimensional conformal radiotherapy was used to treat 19 patients with 2.0-2.75 Gy per daily fraction (median, 
2.5 Gy). Radiotherapy doses ranged from 50 to 66 Gy (median, 55 Gy) in 20-33 fractions over 4-6.5 weeks (Table 1). The most frequently used doses were 66 Gy in 33 fractions (7 patients), 50 Gy in 20 fractions (6 patients), and 55 Gy in 22 fractions (3 patients). Two patients with large BCC ( $2.5 \mathrm{~cm}, \mathrm{cT} 2 \mathrm{NO})$ were treated with $55 \mathrm{~Gy}$ in 22 fractions. One patient with a 0.8 $\mathrm{cm}$ BCC was treated with 50 Gy in 20 fractions; when the patient still had a persistent plaque 6 weeks after completion of radiotherapy, boost electron beam therapy of 5 Gy in 2 fractions was added. An 88-year-old woman with a $1.5-\mathrm{cm}$ SCC had difficulty visiting hospital as frequently as required, so a mildly hypofractionated schedule of $55 \mathrm{~Gy}$ in 20 fractions was applied (2.75 Gy per daily fraction). One patient with SCC received postoperative radiotherapy to a dose of $60 \mathrm{~Gy}$ in 30 fractions due to microscopic residual disease after surgical excision. No patients underwent elective nodal treatment.

For each patient, the electron beam energy (range, 6 to 9 $\mathrm{MeV}$ ) or photon beam energy (6 MV) was chosen based on the estimated tumor depth [2]. A median 5-mm-thickness (range, 3 to $10 \mathrm{~mm}$ ) bolus was used in all patients to ensure the skin surface dose.

For patients treated with electron beam therapy, the energy was selected based on delivering the treatment to the $90 \%$ isodose line [10]. The source-to-surface distance was $100 \mathrm{~cm}$ to the bolus. Various cone sizes $(3-10 \mathrm{~cm})$ were used to protect the surrounding normal organs. A lead shield was placed under the eyelid $(n=1)$, under the cheek $(n=1)$, or under the upper lip $(n=1)$ to minimize normal-tissue toxicity.

\section{Treatment response}

The follow-up time was defined as the time from the completion of radiotherapy until the date of the most recent follow-up visit or death. Local failure was defined as a clinical or radiographic increase in the size of the primary tumor or regional lymph node. LFFS was defined as the time interval from the completion of radiotherapy to the date of local failure, death, or the last follow-up visit.

Patients were evaluated 1 month after the completion of radiotherapy and every 2-3 months for the first 2 years, every 6 months for up to 5 years, and annually thereafter. Patient evaluation included a physical examination and toxicity assessment. Patients were evaluated weekly during EBRT. Acute and late toxicities were reported during both treatment and follow-up visits. Acute toxicities were defined as those occurring within 3 months of radiotherapy completion. Toxicity was scored using the Common Terminology Criteria for Adverse Events (CTCAE) version 4.0. Clinical response was assessed 3 months after radiotherapy according to the Response Evaluation Criteria in Solid Tumors (RECIST) criteria [15].

Cosmetic results were assessed by the physician at each follow-up visit using a standardized cosmetic scale [16]. 'Excellent' was defined as no to slight atrophy, pigment change, hair loss, induration, and loss of subcutaneous fat; 'good' was defined as patch atrophy, moderate telangiectasia, total hair loss, moderate but asymptomatic fibrosis, and/or slight field contracture with less than 10\% linear reduction; 'fair' was defined as marked atrophy and gross telangiectasia, severe induration or loss of subcutaneous tissue, and field contracture greater than 10\% linear measurement; and 'poor' was defined as ulceration or necrosis. The final cosmetic outcome at last follow-up visit was considered for statistical analysis [17].

\section{Statistical analysis}

Statistical analysis was performed using the IBM SPSS version 22.0 software package (IBM SPSS Inc., Armonk, NY, USA). The Kaplan-Meier method was used to determine the LFFS rate. Age refers to the patient's age at the time of radiotherapy.

\section{Results}

\section{Patient characteristics}

The patient, tumor, and treatment characteristics are listed in Table 1. The median age was 75.5 years (range, 52.6 to 92.5 years). The median follow-up duration from the completion of radiotherapy was 44.9 months (range, 5.8 to 82.6 months). The median tumor size was $1.5 \mathrm{~cm}$ (range, 0.5 to $5.2 \mathrm{~cm}$ ).

\section{Treatment outcome}

At the time of analysis, all patients $(n=19)$ were alive 5.8 to 82.6 months after radiotherapy (median, 44.9 months). All the patients ( $n=19,100 \%)$ showed a complete response by a median of 3.0 months after radiotherapy (range, 1.0 to 19.3 months). The 3-year LFFS rate was $91.7 \%$ (Fig. 1). Local recurrence was noted in 1 patient (5.3\%) 32.5 months after radiotherapy (Fig. 2). Salvage operation was performed in this patient. Initially, this patient preferred radiotherapy to surgery because he had diabetes. None of the patients developed metastasis in the lymph nodes or distant organs. None of the patients died from skin cancer during the follow-up. Photos of representative patients are shown in Fig. 3.

\section{Acute and late toxicities}

https://doi.org/10.3857/roj.2019.0038 
Table 1. Patients' characteristics $(n=19)$

\begin{tabular}{|c|c|c|}
\hline Characteristic & & Value \\
\hline Age (yr) & 75.5 & $(52.6-92.5)$ \\
\hline$<65$ & 4 & $(21.0)$ \\
\hline $65-80$ & 9 & $(47.4)$ \\
\hline$>80$ & 6 & $(31.6)$ \\
\hline \multicolumn{3}{|l|}{ Gender } \\
\hline Male & 7 & $(36.8)$ \\
\hline Female & 12 & $(63.2)$ \\
\hline Race, Asian & 19 & $(100)$ \\
\hline \multicolumn{3}{|l|}{ ECOG performance status } \\
\hline$\leq 2$ & 17 & $(89.5)$ \\
\hline$>2$ & 2 & $(10.5)$ \\
\hline \multicolumn{3}{|l|}{ Location of tumor } \\
\hline Cheek & 9 & $(47.3)$ \\
\hline Nose & 5 & $(26.3)$ \\
\hline Periorbital & 2 & $(10.5)$ \\
\hline Forehead & 1 & $(5.3)$ \\
\hline Ear & 1 & $(5.3)$ \\
\hline Neck & 1 & $(5.3)$ \\
\hline \multicolumn{3}{|l|}{ Histology } \\
\hline Squamous cell in situ & 1 & $(5.2)$ \\
\hline Squamous cell & 9 & $(47.4)$ \\
\hline Basal cell & 9 & $(47.4)$ \\
\hline Tumor size $(\mathrm{cm})$ & 1.5 & $(0.5-5.2)$ \\
\hline \multicolumn{3}{|l|}{ Tumor multiplicity } \\
\hline Yes & 3 & $(15.8)$ \\
\hline No & 16 & $(84.2)$ \\
\hline \multicolumn{3}{|l|}{ Tumor stage } \\
\hline Tis & 1 & $(5.3)$ \\
\hline $\mathrm{T} 1(\leq 2 \mathrm{~cm})$ & 11 & $(57.9)$ \\
\hline $\mathrm{T} 2(2-5 \mathrm{~cm})$ & 6 & $(31.5)$ \\
\hline T3 $(>5 \mathrm{~cm})$ & 1 & $(5.3)$ \\
\hline \multicolumn{3}{|l|}{ Lymph node metastasis } \\
\hline No & 19 & $(100)$ \\
\hline \multicolumn{3}{|l|}{ Indication for RT } \\
\hline Primary treatment & 18 & $(94.7)$ \\
\hline Adjuvant treatment & 1 & $(5.3)$ \\
\hline \multicolumn{3}{|l|}{ Treatment beam energy } \\
\hline Electrons (6 MeV) & 4 & $(21.1)$ \\
\hline Electrons (9 MeV) & 11 & $(57.8)$ \\
\hline Photons (6 MV) & 4 & $(21.1)$ \\
\hline \multicolumn{3}{|l|}{ Total dose of RT (Gy) } \\
\hline 55 (2.75 Gy/Fx) & 1 & $(5.3)$ \\
\hline $55(2.5 \mathrm{~Gy} / \mathrm{Fx})$ & 3 & $(15.8)$ \\
\hline $50(2.5 \mathrm{~Gy} / \mathrm{Fx})$ & 6 & $(31.5)$ \\
\hline 64 (2 Gy/Fx) & 1 & $(5.3)$ \\
\hline $66(2 \mathrm{~Gy} / \mathrm{Fx})$ & 7 & $(36.8)$ \\
\hline 60 (2 Gy/Fx), adjuvant & 1 & $(5.3)$ \\
\hline
\end{tabular}

Values are presented as median (range) or number (\%). ECOG, Eastern Cooperative Oncology Group; RT, radiotherapy; Fx, fraction.

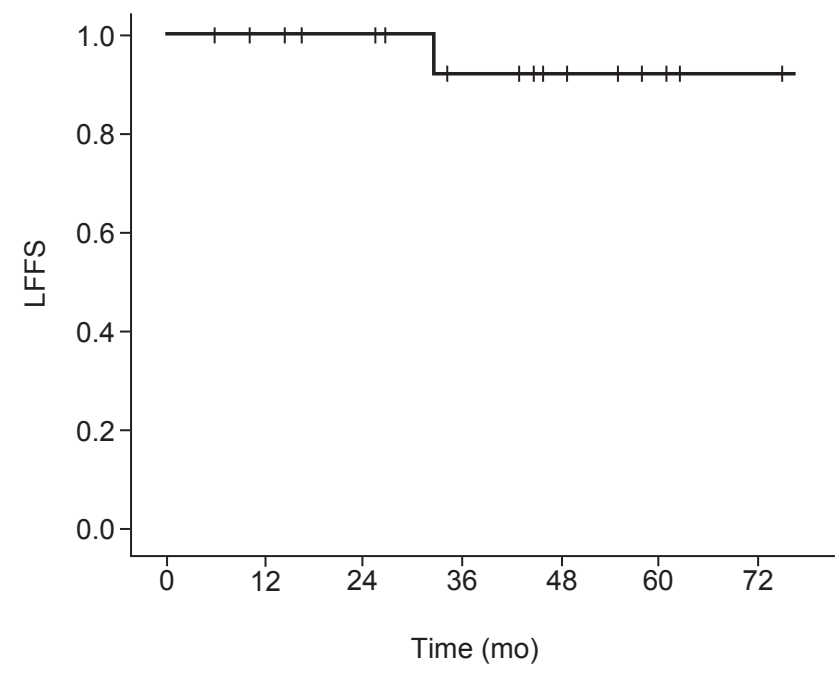

Fig. 1. Local failure-free survival (LFFS) rate after external beam radiotherapy.
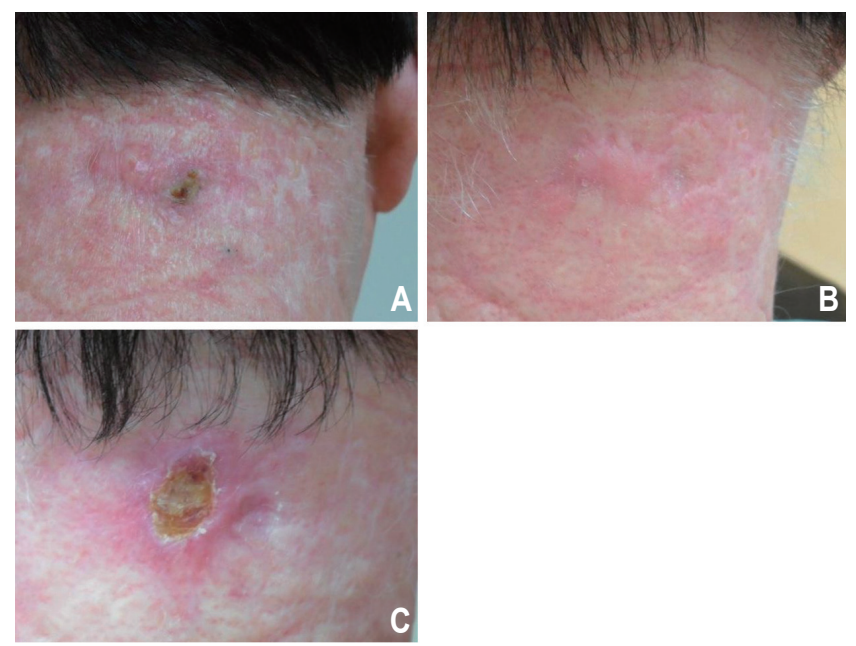

Fig. 2. A 60-year-old man with a 2-cm squamous cell carcinoma of the posterior neck before (A), 3 months (B), and 32.5 months (C) after $50-G y$ radiation therapy in 20 fractions.

The incidences of acute and late toxicity with radiotherapy are shown in Table 2. No grade 3 or higher toxicities were evident. The most common late adverse events were mild hyperpigmentation (5.3\%) and dry skin (10.5\%).

\section{Cosmetic outcomes}

All patients had a cosmetic rating of good or excellent at the last follow-up visit. The cosmetic outcomes were excellent for $16(84.2 \%)$ and good for $3(15.8 \%)$ of the 19 patients. 

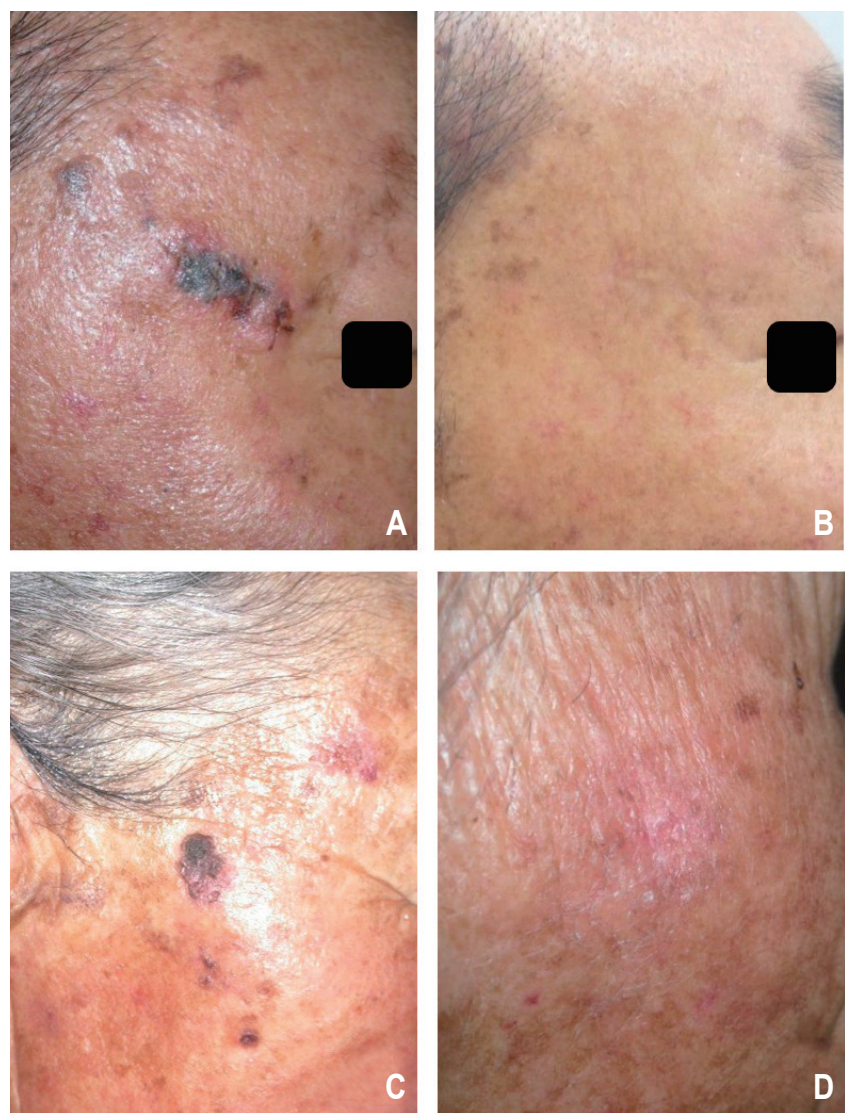

Fig. 3. A 64-year-old man with a 2-cm basal cell carcinoma of the right temple before (A) and 3 years after (B) 66-Gy radiation therapy in 33 fractions. A 90-year-old woman with a 2 -cm squamous cell carcinoma of the right temple before $(C)$ and 3 months after (D) 50-Gy radiation therapy in 20 fractions.

\section{Discussion and Conclusion}

EBRT is an alternative to surgery for patients with primary basal and squamous cell skin cancers in the head and neck region who are not suitable for surgery [8]. Our findings confirm the efficacy and safety of EBRT as a non-surgical treatment option in patients with basal and squamous cell skin cancers in the head and neck region, especially in the Asian population. Our 3-year LFFS rate was 91.7\% (Fig. 1), which was similar to that previously reported for radiotherapy [8] and comparable to data regarding the surgical treatment of basal and squamous cell skin cancers in the head and neck region [18].

Treatment options for BCC and SCC skin cancers in the head and neck region include surgical excision and radiotherapy. Patient factors (age, comorbidity, patient preference, and performance status), tumor factors (size, location, and
Table 2. Incidence of acute and late toxicity $(n=19)$

\begin{tabular}{ll}
\hline \multicolumn{1}{c}{ Toxicity } & No. of patients (\%) \\
\hline Acute (within 3 mo) & $2(10.5)$ \\
Alopecia G1 & $3(15.8)$ \\
Hyperpigmentation G1 & $7(36.8)$ \\
Desquamation G1 (dry) & $2(10.5)$ \\
Desquamation G2 (moist) & $5(26.3)$ \\
Erythema G1 & $3(15.8)$ \\
Pruritus G1 & $1(5.3)$ \\
Oral mucositis G1 & $2(10.5)$ \\
Oral mucositis G2 & $1(5.3)$ \\
Conjunctivitis G1 & \\
Late (>3 mo) & $1(5.3)$ \\
Alopecia G1 & $1(5.3)$ \\
Hyperpigmentation G1 & $1(5.3)$ \\
Hypopigmentation G1 & $1(5.3)$ \\
Pruritus G1 & $2(10.5)$ \\
Dry skin G1 & $1(5.3)$ \\
Oral mucositis G2 &
\end{tabular}

$G$ grade.

thickness), and treatment factors (cure and complication rates and cosmetic and functional results) are considered when selecting the optimal treatment modality [19]. Surgery options include Mohs micrographic technique or wide local excision under general anesthesia with or without flap/graft reconstruction [20]. Jin et al. [21] published a study of 86 patients (94 lesions) with BCC or SCC of the face treated with surgery, obtaining a 96\% local control rate (Table 3). Cosmetic outcomes were mostly excellent or good. Kang et al. [18] reported 120 patients with BCC or SCC of the face treated with surgery, obtaining a $98 \%$ local control rate.

Radiotherapy is an important primary treatment option to preserve anatomy and also in elderly patients unfit for surgery [22]. Palliative radiotherapy is an important modality in patients with advanced and/or incurable disease [20]. In cases of lesions in the ear, nose, lower eyelid, or medial canthus, radiotherapy is favored [20]. In elderly patients with multiple comorbidities, a considerable risk can be associated with general anesthesia [23]. In our study, the median age was 75.5 years (range, 52.6 to 92.5 years). Six patients were older than 80 years $(31.6 \%)$. Radiotherapy can also be performed as an adjuvant to treat gross residual disease or microscopic residual disease after surgery, to reduce the risk of recurrence when reexcision is not possible [24]. Some authors recommend surgery instead of radiotherapy due to cosmetic results [5]. However, 


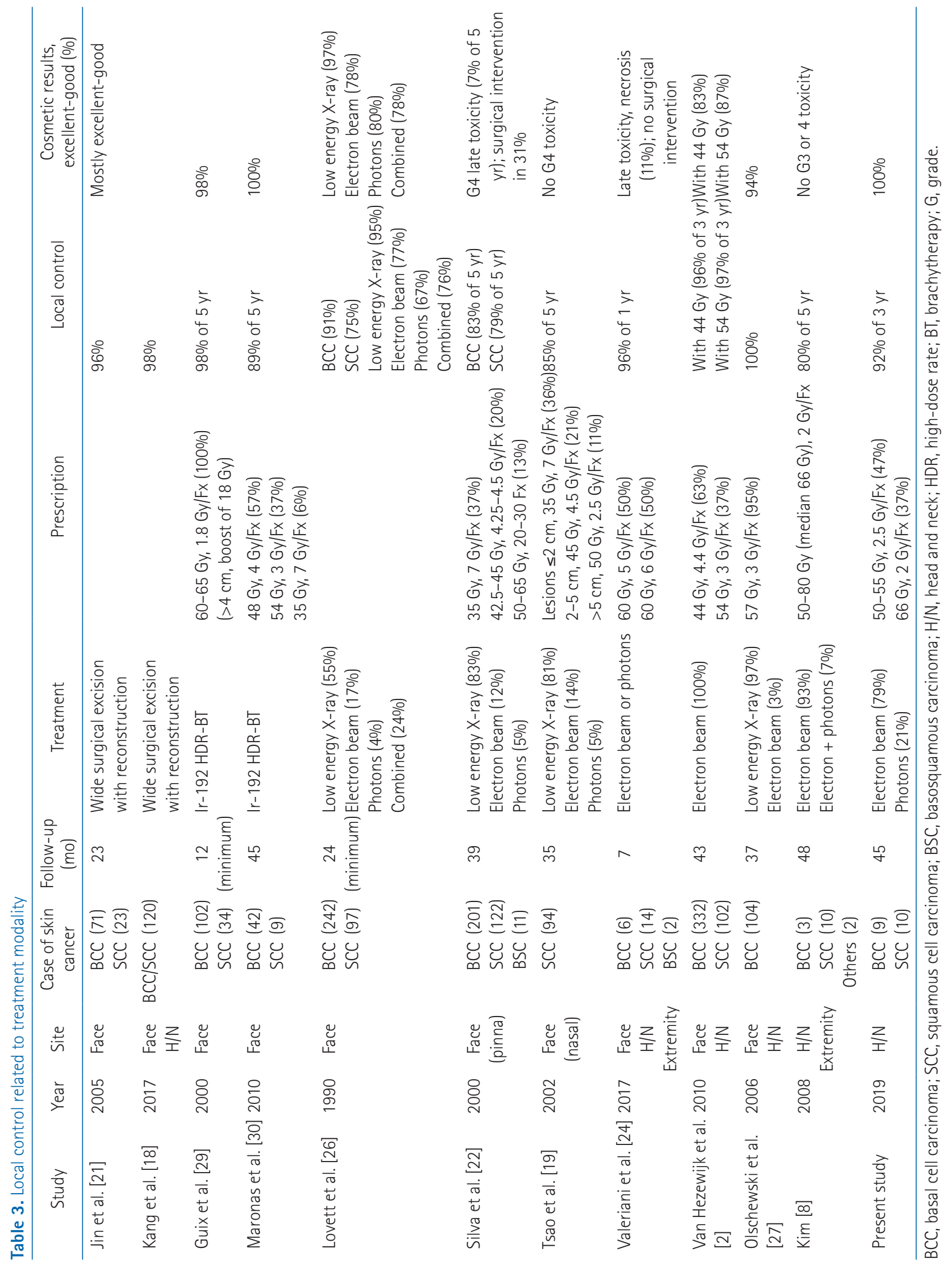


our study showed a good (15.8\%) or an excellent (84.2\%) cosmetic outcome, as scored by the physician. Other groups have also described favorable cosmetic results in patients with radiotherapy $[8,10]$.

BCC and SCC are usually treated with low-energy (kV) $X$-rays or electrons from a linear accelerator, due to their limited penetration. High-energy (MV) X-rays can be used in cancers with deep extension $[8,25,26]$ (Table 3 ). The delivery of low-energy $\mathrm{X}$-rays (100-250 kVp) by a superficial/orthovoltage machine avoids skin sparing and the unnecessary irradiation of deeper tissues $[20,27]$. However, low-energy X-rays are not available at many institutions in our country. Electron beam radiotherapy provides equivalent local control rates but requires careful attention to ensure adequate target coverage and minimize dosimetric variance [28]. The local control rates and cosmetic results are comparable among radiotherapy modalities [19,22].

Patients with BCC or SCC are often treated by highdose rate (HDR) brachytherapy using radioactive isotopes such as iridium-192 with skin surface applicators or surface molds. To be suitable for brachytherapy, lesions should be superficial (<3-4 mm in depth) [20]. Guix et al. [29] evaluated 136 patients with BCC or SCC of the face treated with HDR brachytherapy to a total dose of 60-65 Gy in 33 to 36 fractions (1.8 Gy per fraction) (Table 3). Patients with lesions 4 $\mathrm{cm}$ or more had a boost of $18 \mathrm{~Gy}$ in 10 fractions, after a rest period of 3 weeks, with the total dose ranging from 75 to 80 Gy. The 5 -year local control rate was $98 \%$, and $98 \%$ of the patients had good or excellent cosmesis. Maronas et al. [30] reported a study of 51 patients with BCC or SCC of the face treated with HDR brachytherapy to a total dose of 44-48 Gy in 11 to 12 fractions (57\%) or 51-57 Gy in 17 to 18 fractions (37\%). In three very old patients (6\%), a total dose of $35 \mathrm{~Gy}$ in 5 fractions was administered. The 5 -year local control rate was $89 \%$. Cosmetic results were good or very good in all cases.

A hypofractionated schedule was proposed as a valuable option for elderly disabled patients with small tumors to minimize the number of hospital visits [2]. In our study, 9 patients (47.4\%) were treated with 50-55 Gy in 20-22 fractions (2.5 Gy per daily fraction). One patient (5.3\%) was treated with 55 Gy in 20 fractions (2.75 Gy per daily fraction). More intensive hypofractionated schedules (54 Gy in 18 fractions and $44 \mathrm{~Gy}$ in 10 fractions, four times per week) were reported with good local control and cosmetic outcomes [2] (Table 3). Tsao et al. [19] published a series of 94 patients with SCC of the nasal skin treated with EBRT, obtaining an 85\% 5 -year local control rate with no grade 4 toxicity. In general, short fractionation regimens (e.g., 35 Gy in 5 fractions) were used for small lesions $(\leq 2 \mathrm{~cm})$ and elderly patients who did not wish to attend a longer course of treatment. Valeriani et al. [24] reported schedules of 2 fractions per week (60 Gy in 12 fractions and 60 Gy in 10 fractions) in very elderly patients. Late toxicity, represented by necrosis, occurred in 3 cases $(11 \%)$, but none needed surgical intervention. Silva et al. [22] published a series of 334 patients with skin cancer of the pinna treated with EBRT. The most frequently used dose prescriptions were $35 \mathrm{~Gy}$ in 5 fractions (123 treatments with median field size $=4.9 \mathrm{~cm}^{2}$ ). The 5 -year actuarial rate of significant grade 4 late toxicity was 7.3\% (19 patients). Six patients required surgical intervention. The local control rates of hypofractionated schedules were comparable to those of conventional schedules $[19,22,24]$. However, there were several severe late toxicities in some reports [22,24]. Silva et al. [22] suggested that dose-fractionation schedules using fraction sizes $<4$ Gy may reduce the risk of necrosis and ulceration, particularly for field sizes $>5 \mathrm{~cm}^{2}$. Veness and Richards [20] reported that smaller treatment fields $(2-3 \mathrm{~cm})$ tolerate hypofractionation better than larger areas, but if cosmesis is important, larger fractions should still be avoided. Tsao et al. [19] reported that most patients treated with hypofractionated schedules ( $<4$ weeks) will have a visible cosmetic defect $>1-2$ years after radiotherapy completion.

Thanh Pham et al. [9] reported that complete tumor regression after treatment may take up to 2 to 3 months, and early biopsies may be misleading secondary to atypical fibroblasts induced by the treatment. In our study, we observed complete tumor regression at a median of 3.0 months after radiotherapy (range, 1.0-19.3 months). Ten patients (52.6\%) showed a complete response at or later than 3.0 months after radiotherapy. One patient with BCC still had a pigmented lesion at the 4-month follow-up, and the biopsy of this lesion at the 5-month follow-up revealed no evidence of malignancy. The pigmented lesion disappeared at 19 months after radiotherapy. In selected cases, watchful waiting up to 3 months or more after radiotherapy can be a reasonable approach.

The limitations of our study are its low sample size, retrospective nature, and short follow-up period. We could not analyze the important prognostic factors for local control, such as histology (SCC and BCC) and tumor size.

In conclusion, we confirmed that EBRT is an effective and safe treatment option in patients with basal and squamous cell skin cancers in the head and neck region, especially in elderly Asian patients. Our results also suggest that watchful waiting up to 3 months or more after radiotherapy can be a reasonable 
approach in selected cases.

\section{Conflict of Interest}

No potential conflict of interest relevant to this article was reported.

\section{Acknowledgments}

This work was supported by the research grant of Jeju National University in 2016.

\section{References}

1. Haseltine JM, Parker M, Wernicke AG, Nori D, Wu X, Parashar B. Clinical comparison of brachytherapy versus hypofractionated external beam radiation versus standard fractionation external beam radiation for non-melanomatous skin cancers. J Contemp Brachytherapy 2016;8:191-6.

2. van Hezewijk $M$, Creutzberg $C L$, Putter $H$, et al. Efficacy of a hypofractionated schedule in electron beam radiotherapy for epithelial skin cancer: analysis of 434 cases. Radiother Oncol 2010;95:245-9.

3. Kim HS, Cho EA, Bae JM, et al. Recent trend in the incidence of premalignant and malignant skin lesions in Korea between 1991 and 2006. J Korean Med Sci 2010;25:924-9.

4. Leibovitch I, Huilgol SC, Selva D, Hill D, Richards S, Paver R. Cutaneous squamous cell carcinoma treated with Mohs micrographic surgery in Australia. I: Experience over 10 years. J Am Acad Dermatol 2005;53:253-60.

5. Petit JY, Avril MF, Margulis A, et al. Evaluation of cosmetic results of a randomized trial comparing surgery and radiotherapy in the treatment of basal cell carcinoma of the face. Plast Reconstr Surg 2000;105:2544-51.

6. Veness MJ. The important role of radiotherapy in patients with non-melanoma skin cancer and other cutaneous entities. J Med Imaging Radiat Oncol 2008;52:278-86.

7. Veness M. Hypofractionated radiotherapy in older patients with non-melanoma skin cancer: less is better. Australas J Dermatol 2018;59:124-7.

8. Kim JH. Radiation therapy in elderly skin cancer. J Korean Soc Ther Radiol Oncol 2008;26:113-7.

9. Thanh Pham T, Cross S, Gebski V, Veness MJ. Squamous cell carcinoma of the lip in Australian patients: definitive radiotherapy is an efficacious option to surgery in select patients. Dermatol Surg 2015;41:219-25.

10. Ferro M, Deodato F, Macchia G, et al. Short-course radiotherapy in elderly patients with early stage nonmelanoma skin cancer: a phase II study. Cancer Invest 2015;33:34-8.

11. Bhatnagar A, Loper A. The initial experience of electronic brachytherapy for the treatment of non-melanoma skin cancer. Radiat Oncol 2010;5:87.

12. Nguyen NP, Ries T, Vock J, et al. Effectiveness of radiotherapy for elderly patients with non-melanoma skin cancer of the head. Geriatr Gerontol Int 2015;15:601-5.

13. Bhatnagar A. Nonmelanoma skin cancer treated with electronic brachytherapy: results at 1 year. Brachytherapy 2013;12:134-40.

14. Gauden R, Pracy M, Avery AM, Hodgetts I, Gauden S. HDR brachytherapy for superficial non-melanoma skin cancers. J Med Imaging Radiat Oncol 2013;57:212-7.

15. Therasse $P$, Arbuck SG, Eisenhauer EA, et al. New guidelines to evaluate the response to treatment in solid tumors. J Natl Cancer Inst 2000;92:205-16.

16. Cox JD, Stetz J, Pajak TF. Toxicity criteria of the Radiation Therapy Oncology Group (RTOG) and the European Organization for Research and Treatment of Cancer (EORTC). Int J Radiat Oncol Biol Phys 1995;31:1341-6.

17. Pampena R, Palmieri T, Kyrgidis $A$, et al. Orthovoltage radiotherapy for nonmelanoma skin cancer (NMSC): comparison between 2 different schedules. J Am Acad Dermatol 2016;74:341-7.

18. Kang JK, Yun BM, Song JK, Shin MS. Non-melanocytic skin cancers of the head and neck: a clinical study in Jeju Province. Arch Plast Surg 2017:44:313-8.

19. Tsao MN, Tsang RW, Liu FF, Panzarella T, Rotstein L. Radiotherapy management for squamous cell carcinoma of the nasal skin: the Princess Margaret Hospital experience. Int $J$ Radiat Oncol Biol Phys 2002;52:973-9.

20. Veness M, Richards S. Role of modern radiotherapy in treating skin cancer. Australas J Dermatol 2003;44:159-66.

21. Jin $H R$, Lee $J Y$, Lee $D W$, et al. Primary facial skin cancer: clinical characteristics and surgical outcome in Chungbuk Province, Korea. J Korean Med Sci 2005;20:279-82.

22. Silva JJ, Tsang RW, Panzarella T, Levin W, Wells W. Results of radiotherapy for epithelial skin cancer of the pinna: the Princess Margaret Hospital experience, 1982-1993. Int J Radiat Oncol Biol Phys 2000;47:451-9.

23. Momm F, Becker G, Bartelt S, Guttenberger R. The elderly, fragile tumor patient: radiotherapy as an effective and most feasible treatment modality. J Pain Symptom Manag 2004;27:3-4.

24. Valeriani M, Nicosia L, Agolli L, et al. Mono- and bi-weekly 
hypofractionated radiation therapy for the treatment of epithelial skin cancer in very elderly patients. Anticancer Res 2017;37:825-30.

25. Newlands C, Currie R, Memon A, Whitaker S, Woolford T. Non-melanoma skin cancer: United Kingdom National Multidisciplinary Guidelines. J Laryngol Otol 2016;130(S2):S125-S132.

26. Lovett RD, Perez CA, Shapiro SJ, Garcia DM. External irradiation of epithelial skin cancer. Int J Radiat Oncol Biol Phys 1990;19:235-42.

27. Olschewski T, Bajor K, Lang B, Lang E, Seegenschmiedt MH. Radiotherapy of basal cell carcinoma of the face and head: Importance of low dose per fraction on long-term outcome. J
Dtsch Dermatol Ges 2006;4:124-30.

28. Rong Y, Zuo L, Shang L, Bazan JG. Radiotherapy treatment for nonmelanoma skin cancer. Expert Rev Anticancer Ther 2015;15:765-76.

29. Guix B, Finestres F, Tello J, et al. Treatment of skin carcinomas of the face by high-dose-rate brachytherapy and custommade surface molds. Int J Radiat Oncol Biol Phys 2000;47:95102.

30. Maronas M, Guinot $J$, Arribas L, et al. Treatment of facial cutaneous carcinoma with high-dose rate contact brachytherapy with customized molds. Brachytherapy 2011;10:221-7. 